\title{
Les régulations locales en classe de FLE
}

\section{Résumé :}

La réalisation d'un dispositif didactique implique une régulation continue pour la construction et les transformations de l'objet enseigné. Les interactions didactiques sont le lieu d'une régulation permanente qui se réalise souvent par la désignation d'une dimension de l'objet non perçue par les élèves ou par le traitement d'un obstacle qui bloque la construction d'un savoir.

Nous essayerons dans cet article de répondre à la question suivante: comment les enseignants régulent-ils les apprentissages dans les séances de grammaire et quels sont les obstacles régulés par ces derniers ? Y a-t-il vraiment régulation de leur part ?

Mots- clés : pratiques enseignantes- régulation objet enseigné- interaction didactique.

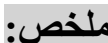

إن إنجاز جهاز للتعليمية يستدعي تتظيما متو اصلا من أجل بناء و تحويلات الموضوع الذي يتم تدريسه. إن التداخلات التعليمية هي مكان تنظيم مستمر يتحقق غالبا عن طريق تعيين

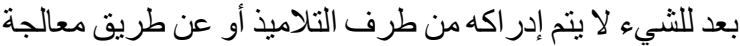
عقبة تعيق بناء معرفة ماء. سنحاول في هذا المقال الإجابة على السؤال التالي: كيف يقوف مأل



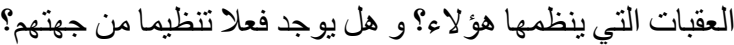

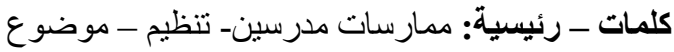
مدرس - تفاعل تعليمي

\section{Camélia Nabila Bechiri}

Département de Française Université du 20 Août 1955

Skikda

\section{Introduction :}

Dans cet article, nous nous intéressons au deuxième geste professionnel de l'enseignant: celui de régulation. Ce concept fait partie des théories centrées sur le processus d'apprentissage, il a été adapté à un cadre didactique dans lequel l'objet d'enseignement occupe une place décisive. La réalisation d'un dispositif didactique implique une régulation continue pour la construction et les transformations de l'objet enseigné. 
Schneuwly (2009, p.136) conçoit le geste de régulation comme la prise d'informations en classe et l'interprétation de ces informations, ainsi que les outils employés à cette fin. La régulation, fondée sur des critères implicites et explicites, contribue de manière cruciale à la construction de l'objet enseigné. Les gestes de régulation dans les interactions didactiques concernent l'ensemble des processus d'enseignement et d'apprentissage. Les interactions didactiques sont le lieu d'une régulation permanente qui se réalise souvent par la désignation d'une dimension de l'objet non perçue par les élèves ou par le traitement d'un obstacle qui bloque la construction d'un savoir.

Nous essayerons dans cet article de répondre à la question suivante : comment les enseignants régulent-ils les apprentissages dans les séances de grammaire et quels sont les obstacles régulés par ces derniers ? Y a-t-il vraiment régulation de leur part?

Nous tenons à montrer dans un premier temps quels sont les principaux types de dispositifs régulés. Puis, dans un deuxième temps, nous allons voir quels sont les principaux obstacles visibles dans la construction de l'objet « grammaire ».

\section{Définition}

Dans le déroulement du travail enseignant en classe, nous en distinguons deux formes : les « régulations internes », les « régulations locales » (Schneuwly et Bain, 1993).

- Régulations internes : démarches outillées pour l'enseignant et l'élève.

- Régulations locales : interactions en continu contribuant à la construction de l'objet.

Nous retenons que, par le geste de régulation, l'enseignant adapte les dispositifs didactiques mis en œuvre et reconfigure son action didactique en prenant en compte plusieurs facteurs.

Selon Schneuwly, pour réaliser un dispositif didactique, il faut une régulation continue qui assure la construction et la transformation de l'objet enseigné. Le geste de régulation surgit lorsqu'un écart apparaît entre un attendu et un réel perçu (Barbier, 1985, cité dans Saussez \& Allal, 2007) et la prise en considération de cet écart conduit à une prise de décision de régulation.

L'on peut observer la régulation du processus d'enseignement/apprentissage selon différents points de vue et cette régulation s'opère à différents niveaux. Il $\mathrm{y}$ a régulation interne et régulation locale.

Schneuwly et Dolz (2009) définissent comme la régulation interne une régulation qui fait objet de démarches outillées et sert à la récolte d'informations sur l'état des connaissances des élèves. 


\section{Quand l'auteur se livre : du vécu à la création littéraire Notes d'une musique ancienne de Salah Benlabed}

Les régulations internes peuvent se situer en début, pendant ou à la fin d'un cycle d'apprentissage. Quand la régulation interne est placée avant les tâches et se servant des productions initiales d'élèves, elle permet « d'adapter la séquence aux capacités d'une classe donnée et garantit ainsi la cohérence interne d'une séquence ». Insérée dans des tâches, elle peut utiliser des textes authentiques qui «constituent l'interface indispensable avec les connaissances sur le genre étudié ». Située à la fin, elle est dotée d'outils synthétisant « sous forme explicite les résultats des observations, analyses et exercices et sert de régulateur de l'activité propre de l'élève » (Schneuwly et Bain, 1993, p.221).

La régulation locale s'effectue durant la réalisation des activités scolaires à la faveur d'un échange avec l'élève. Elle est liée aux interactions entre les trois pôles du triangle didactique. La centration sur les régulations locales implique de décrire des formes interactives qui contribuent à la construction de l'objet enseigné.

L'étude des régulations doit permettre de comprendre et de décrire la dynamique de la construction de l'objet enseigné en classe de français en Algérie. L'analyse des régulations à l'intérieur d'une leçon ou d'une séquence permet donc de cibler des interactions qui peuvent conduire à la construction de l'objet enseigné (grammaire). Ainsi, son analyse fournit des informations essentielles sur la manière dont l'objet se construit, les obstacles observés par l'enseignant ou exprimés par l'élève ou encore les apports pour réguler l'apprentissage de l'élève dans l'action. Face à ces informations, l'enseignant doit réagir; ses réactions constituent alors un moment de régulation.

Dans cette intervention, nous tenons à montrer que l'objet enseigné peut s'appréhender aussi au travers des obstacles vécus dans les leçons par l'enseignant et les élèves et au travers de gestes de l'enseignant que nous analyserons.

Nous pouvons considérer dans notre travail les reformulations d'une consigne par l'enseignant comme étant une forme de régulation.

Dans notre article, nous nous focalisons sur les régulations locales, cela signifie décrire des formes interactives qui peuvent contribuer ou au contraire entraver la construction et les transformations de l'objet d'enseignement/apprentissage. C'est une analyse fine qui suit la construction des objets d'enseignement pas à pas.

La centration sur les régulations locales suppose de décrire des formes interactives qui contribuent à la construction à la fois de l'objet d'enseignement et des manières d'en parler communes à la classe.

Le nombre de régulations locales est très élevé. Nous pouvons dire que de grandes parties des leçons d'enseignements en sont constituées. Le repérage d'extraits significatifs des multiples occurrences des objets grammaticaux est effectué au moyen des critères suivants. 
- L'extrait correspond à un échange entre enseignant et élèves à propos de la dimension retenue lors d'un feedback, d'un exercice, d'une question.

- L'extrait est délimité soit par le support matériel (dans le cadre d'un même exercice, le fait de passer à un autre item marque les limites de l'extrait), soit par un même thème conversationnel (le fait de passer à une intervention portant sur d'autres dimensions de l'objet ne présentant pas de liens avec ce qui a été traité conduit aussi à considérer qu'il y a deux extraits).

1 À la recherche des régulations dans les séances observées

\subsection{Les types de dispositifs régulés}

Dans notre recherche, nous n'allons pas prendre en considération les échanges interactifs élèves-élèves. Nous focalisons notre observation sur les échanges interactifs entre enseignants et élèves à propos de l'objet à construire. Chaque fois que l'enseignant intervient ou réagit aux conduites des apprenants qui agissent dans le dispositif mis en place, il y a régulation.

Nous allons répertorier les régulations des enseignants en fonction des types de dispositifs mis en œuvre. 
Quand l'auteur se livre : du vécu à la création littéraire

Notes d'une musique ancienne de Salah Benlabed

Tableau : Les dispositifs didactiques régulés

\begin{tabular}{|c|c|c|c|c|c|c|c|c|}
\hline $\begin{array}{l}\text { Type } \\
\text { de } \\
\text { dispos } \\
\text { itif }\end{array}$ & $\begin{array}{l}\text { Gram } \\
\text { maire } \\
\text { monsi } \\
\text { eurT } \\
\text { N.évé } \\
\text { n. } \\
\text { régul } \\
\text { atifs }\end{array}$ & $\begin{array}{l}\text { Gram } \\
\text { maire } \\
\text { mada } \\
\text { meA } \\
\text { N.évé } \\
\text { n. } \\
\text { régul } \\
\text { atifs }\end{array}$ & $\begin{array}{l}\text { Gram } \\
\text { maire } \\
\text { mada } \\
\text { meH } \\
\text { N.évé } \\
\text { n. } \\
\text { régul } \\
\text { atifs }\end{array}$ & $\begin{array}{l}\text { Gram } \\
\text { maire } \\
\text { mada } \\
\text { meO } \\
\text { N.évé } \\
\text { n. } \\
\text { régul } \\
\text { atifs }\end{array}$ & $\begin{array}{l}\text { Vocab } \\
\text { ulaire } \\
\text { monsi } \\
\text { eurB } \\
\text { N.évé } \\
\text { n. } \\
\text { régula } \\
\text { tifs }\end{array}$ & $\begin{array}{l}\text { Vocab } \\
\text { ulaire } \\
\text { monsi } \\
\text { eurB } \\
\text { N.évé } \\
\text { n. } \\
\text { régula } \\
\text { tifs }\end{array}$ & $\begin{array}{l}\text { Ortho } \\
\text { graphe } \\
\text { monsi } \\
\text { eurS } \\
\text { N.évé } \\
\text { n. } \\
\text { régula } \\
\text { tifs }\end{array}$ & $\begin{array}{l}\text { Conju } \\
\text { gaison } \\
\text { monsi } \\
\text { eurG } \\
\text { N.évé } \\
\text { n. } \\
\text { régula } \\
\text { tifs }\end{array}$ \\
\hline $\begin{array}{l}\text { Lectur } \\
\text { e de } \\
\text { texte } \\
\text { ou de } \\
\text { phrase } \\
=11\end{array}$ & 2 & 2 & 0 & $\begin{array}{l}\text { Pas } \\
\text { de } \\
\text { lectur } \\
\text { e }\end{array}$ & $\begin{array}{l}\text { Pas de } \\
\text { lectur } \\
\text { e }\end{array}$ & $\begin{array}{l}\text { Pas de } \\
\text { lectur } \\
\text { e }\end{array}$ & 0 & 7 \\
\hline $\begin{array}{l}\text { Explic } \\
\text { ation } \\
\text { de } \\
\text { l'objet } \\
\text { enseig } \\
\text { ner } \\
\text { (quest } \\
\text { ion - } \\
\text { répons } \\
\text { e)=19 }\end{array}$ & 5 & 2 & 1 & 0 & 0 & 2 & 7 & 2 \\
\hline $\begin{array}{l}\text { Formu } \\
\text { lation } \\
\text { de } \\
\text { règles } \\
=00\end{array}$ & 0 & 0 & 0 & 0 & 0 & 0 & 0 & 0 \\
\hline $\begin{array}{l}\text { Exem } \\
\text { ples } \\
=02\end{array}$ & 1 & 0 & 1 & $\begin{array}{l}\text { Pas } \\
\text { d'exe } \\
\text { mple }\end{array}$ & 0 & $\begin{array}{l}\text { Pas } \\
\text { d'exe } \\
\text { mple }\end{array}$ & $\begin{array}{l}\text { Pas } \\
\text { d'exe } \\
\text { mple }\end{array}$ & 0 \\
\hline $\begin{array}{l}\text { Exerci } \\
\text { ce oral } \\
=02\end{array}$ & $\begin{array}{l}\text { Pas } \\
\text { d'exe } \\
\text { rcice } \\
\text { oral }\end{array}$ & $\begin{array}{l}\quad \text { Pas } \\
\text { d'exe } \\
\text { rcice } \\
\text { oral }\end{array}$ & 0 & 1 & 1 & \begin{tabular}{l}
\multicolumn{1}{c}{ Pas } \\
d'exer \\
cice \\
oral \\
\end{tabular} & $\begin{array}{l}\text { Pas } \\
\text { d'exer } \\
\text { cice } \\
\text { oral }\end{array}$ & $\begin{array}{l}\quad \text { Pas } \\
\text { d'exer } \\
\text { cice } \\
\text { oral }\end{array}$ \\
\hline $\begin{array}{l}\text { Exerci } \\
\text { ce } \\
\text { écrit } \\
=62\end{array}$ & 4 & 04 & $\begin{array}{l}\text { Pas } \\
\text { d'exe } \\
\text { rcice } \\
\text { écrit }\end{array}$ & 28 & 4 & 0 & 19 & 3 \\
\hline
\end{tabular}




\begin{tabular}{|l|l|l|l|l|l|l|l|l|}
\hline $\begin{array}{l}\text { Total } \\
=96\end{array}$ & 12 & 08 & 02 & 29 & 5 & 2 & 26 & 12 \\
\hline
\end{tabular}

Pour les 8 leçons de grammaire filmées, nous avons identifié 96 événements régulatifs, en d'autres termes, tout échange interactif entre maître et élèves, délimité par un changement de matériel, de forme sociale ou de consigne. Nous nous basons également sur les travaux de Bain \& Schneuwly qui conçoivent le geste de régulation «comme la prise d'information, l'interprétation, la correction concernant l'avancement du travail dans les dispositifs didactiques en cours de construction de l'objet enseigné » (1993, p.38) dans les leçons d'enseignement. Les régulations ont comme point de départ le constat d'obstacles rencontrés par les élèves ou des contributions d'élèves à la construction d'objets. Face à ces derniers, l'enseignant doit réagir.

L'analyse des types de dispositifs régulés montre que dans chaque pratique singulière, la plupart des dispositifs mis en œuvre ne font pas l'objet de régulations. Trois dispositifs (formulation de règles - exemples - exercice oral) ne comportent pas ou très peu de régulations car les interventions des élèves sont absentes ou limitées. Les élèves ne prennent pas la parole, ne participent pas à la construction de l'objet. Dans le dispositif didactique formulation de règles, l'enseignant se positionne dans un discours magistral et prend toute la place au détriment des élèves.

Les régulations sont plus fréquentes dans les trois autres dispositifs (lecture de texte - explication de l'objet enseigné - exercice écrit). Ceci s'explique par la nature même des dispositifs qui sollicitent des interventions de la part des élèves. Les régulations locales constituent des formats interactifs favorisant l'intervention des élèves.

Nous avons donc focalisé notre regard plus précisément sur les interactions régulatives à l'intérieur de ces trois dispositifs afin d'analyser les obstacles vécus et comment enseignant et élèves contribuent à la création d'un espace de signification autour de l'objet grammaire.

Nous constatons que la leçon de monsieur « $\mathrm{S} »$ contient un nombre élevé de régulations locales : 26 , nous pensons que cela est dû à la nature même de la leçon « formation d'adverbe ». L'enseignant sollicite beaucoup les élèves et les aide à participer en leur demandant de suivre la règle de formation d'adverbe. Nous pouvons en déduire que lorsque l'élève s'implique dans la leçon par sa participation, il y a plus de régulations de la part de l'enseignant.

La leçon de madame $\mathrm{O}$ contient aussi un nombre élevé de régulations : 29. Cela est dû aux dispositifs didactiques choisis: trois exercices (deux exercices écrits et un oral). 
L'on peut aussi dire que les trois enseignants (monsieur T- madame Amadame $\mathrm{H}$ ) qui enseignent la même leçon (types de phrase) ont utilisé le même outil didactique, ils ont bénéficié d'un même volume horaire, ils ont respecté le programme institutionnel. Toutefois, en ce qui concerne les régulations locales, nous constatons que monsieur $\mathrm{T}$ régule plus (12 régulations) par rapport à madame $\mathrm{A}$ (08 régulations) et madame $\mathrm{H}$ (02 régulations).

Dans la pratique de classe, monsieur T sollicite énormément ses élèves et les incite à participer, alors que madame $\mathrm{H}$ travaille seulement avec la bonne élève de la classe. Les élèves de madame $\mathrm{A}$ ont des difficultés à participer et il $\mathrm{y}$ a un nombre important d'élèves qui perturbe le déroulement de la leçon. L'enseignante est donc dans l'obligation de faire avancer sa leçon même si elle doit poser la question et y répondre elle-même.

En conclusion, nous pouvons dire que lorsque l'enseignant donne la possibilité aux élèves d'échanger leurs idées et de participer, il y a plus de régulation de la part de ce dernier et même parfois de la part des élèves. Car la participation amène l'enseignant à constater les lacunes et les erreurs commises par les élèves. Grâce à cela, l'enseignant peut modifier ou réguler son enseignement.

\section{Les obstacles significatifs dans la construction de l'objet grammaire}

2.1 Obstacles liés à la lecture et compréhension du texte ou de phrases

La lecture du texte ou de la phrase est seulement une étape pour rentrer dans le vif du sujet (la leçon de grammaire). Mais cette étape n'est pas facile car les élèves ont du mal à lire, à prononcer correctement les mots. Dans leur lecture, il y a beaucoup d'hésitations. Les enseignants doivent à chaque fois corriger la prononciation.

Pour ce qui est de la compréhension du texte, certains enseignants (monsieur G-monsieur T) attachent une grande importance à ce dispositif en posant plusieurs questions pour s'assurer que les élèves arrivent à comprendre le texte. Tandis que madame $\mathrm{A}$ et madame $\mathrm{H}$ posent quelques questions qui ne concernent pas la compréhension, ce sont des questions juste pour entamer le cours. Les autres enseignants (monsieur S- monsieur B- madame O) utilisent le texte ou les phrases juste pour extraire les exemples qui les intéressent dans le déroulement de la leçon.

Tous les enseignants filmés ont proposé soit un texte extrait du manuel scolaire soit des phrases tirées du même manuel.

Le dispositif de lecture a fait l'objet d'interactions régulatives, à l'initiative de l'enseignant. Plusieurs d'entre elles ont été mises en œuvre pour 
inciter les élèves à identifier de quoi parle le texte en utilisant les indices du texte à leur disposition et cela en fin de lecture pour comprendre le sens du texte.

Tableau : Obstacles liés à la lecture et la compréhension du texte ou des phrases

\begin{tabular}{|l|l|c|c|}
\hline Types d'obstacles & $\begin{array}{l}\text { Types } \\
\text { d'événements } \\
\text { régulatifs }\end{array}$ & $\begin{array}{l}\text { Nombre } \\
\text { d'événements } \\
\text { régulatifs }\end{array}$ & $\begin{array}{l}\text { Nombre de } \\
\text { leçons }\end{array}$ \\
\hline La lecture & $\begin{array}{l}\text { Régulations } \\
\text { individuelles par } \\
\text { initiées } \\
\text { l'enseignant }\end{array}$ & 11 & $5 / 8$ \\
\hline $\begin{array}{l}\text { Identification des } \\
\text { indices du texte } \\
\text { pour trouver de } \\
\text { quoi parle } \\
\text { l'auteur }\end{array}$ & $\begin{array}{l}\text { Régulations } \\
\text { collectives } \\
\text { initiées } \\
\text { l'enseignant par }\end{array}$ & 04 & $2 / 8$ \\
\hline $\begin{array}{l}\text { Sens d'un mot / } \\
\text { sens du texte }\end{array}$ & $\begin{array}{l}\text { Régulations } \\
\text { individuelles } \\
\text { initiées } \\
\text { l'enseignant par }\end{array}$ & 17 & $4 / 8$ \\
\hline
\end{tabular}

Nous remarquons un aspect très important qui fait l'objet de nombreuses interactions régulatives dans presque la moitié des leçons observées : le «sens d'un mot/sens du texte ». Ce type d'obstacle est plutôt identifié par l'enseignant à travers l'observation de la réaction des apprenants. L'enseignant remarque que son discours n'est pas compréhensible pour eux. Ce qui le pousse à reformuler ses explications en donnant le sens de chaque mot dans le texte. Il essaie de clarifier le sens des mots par leurs équivalents sémantiques en français.

Concernant le type d'obstacle « identification des indices du texte pour trouver de quoi parle l'auteur », deux enseignants ont incité les élèves à identifier de quoi parle le texte.

Enseignants et élèves ont essayé de créer du sens autour de la lecture du texte et d'identifier l'objet enseigné (verbe impératif - types de phrases). Si nous regardons de plus près qui, de l'enseignant ou des élèves, est à l'origine de ces régulations, nous remarquons qu'elles partent toutes d'erreurs commises par les élèves mais sont plutôt à l'initiative de l'enseignant. Cette étape de compréhension du texte est très importante pour préparer les apprenants à l'activité suivante. Mais nous constatons que les autres enseignants n'attachent aucune importance au sens du texte dans les leçons de grammaire. 
Quand l'auteur se livre : du vécu à la création littéraire Notes d'une musique ancienne de Salah Benlabed

2.2 Obstacles liés à l'explication du cours et à la reformulation des règles (lois)

Tableau : Obstacles liés à l'explication du cours et reformulation des règles (lois)

\begin{tabular}{|l|l|c|c|}
\hline $\begin{array}{l}\text { Types } \\
\text { d'obstacles }\end{array}$ & $\begin{array}{l}\text { Types } \\
\text { d'événements } \\
\text { régulatifs }\end{array}$ & $\begin{array}{l}\text { Nombre } \\
\text { d'événements } \\
\text { Régulatifs }\end{array}$ & Enseignants \\
\hline $\begin{array}{l}\text { Explication de } \\
\text { l'objet } \\
\text { grammaire }\end{array}$ & $\begin{array}{l}\text { Régulations } \\
\text { collectives } \\
\text { initiées par } \\
\text { l'enseignant }\end{array}$ & 19 & $8 / 8$ \\
\hline $\begin{array}{l}\text { Formulation de } \\
\text { la règle }\end{array}$ & $\begin{array}{l}\text { L'enseignant } \\
\text { formule les règles } \\
\text { seul. }\end{array}$ & 00 & $8 / 8$ \\
\hline
\end{tabular}

Lors de la construction de l'objet grammaire, les enseignants doivent redéfinir avec d'autres mots certains termes spécifiques comme : types de phrases, champ lexical, synonymie, voix passive et voix passive, l'impératif présent, formation des adverbes, etc. Dans la leçon de types de phrases, les enseignants attirent l'attention des élèves soit sur la ponctuation à la fin de la phrase soit sur l'intonation. Pour ce qui est de la phrase impérative, les enseignants font constater que le verbe n'a pas de sujet.

Dans la leçon sur le champ lexical, l'enseignant essaye d'expliquer chaque mot de sa liste "se désaltérait-courant-onde-breuvage " (extraite du manuel scolaire). En effet, il essaye à chaque fois de glisser le mot « eau » pour que les élèves arrivent à comprendre que le champ lexical se réfère à cette même idée.

\subsection{Obstacles liés à l'exercice écrit}

Les six enseignants observés (à l'exception de madame $\mathrm{H}$ qui ne propose pas aux élèves d'exercice écrit) mettent en place le dispositif didactique (exercice écrit) par la découverte de la consigne ; c'est eux qui lisent à haute voix la consigne extraite du manuel scolaire. 
Tableau : Obstacles liés à l'exercice écrit

\begin{tabular}{|c|l|c|c|}
\hline $\begin{array}{l}\text { Types } \\
\text { d'obstacles }\end{array}$ & $\begin{array}{l}\text { Types } \\
\text { d'événements } \\
\text { régulatifs }\end{array}$ & $\begin{array}{l}\text { Nombre } \\
\text { d'événements } \\
\text { régulatifs }\end{array}$ & Enseignants \\
\hline $\begin{array}{c}\text { Cherche à } \\
\text { résoudre la } \\
\text { consigne de } \\
\text { l'exercice }\end{array}$ & $\begin{array}{l}\text { Régulations } \\
\text { individuelles } \\
\text { initiées par les } \\
\text { élèves }\end{array}$ & 03 & $7 / 8$ \\
\hline $\begin{array}{c}\text { Cherche à } \\
\text { résoudre la } \\
\text { consigne de } \\
\text { l'exercice }\end{array}$ & $\begin{array}{l}\text { Régulations } \\
\text { collectives } \\
\text { initiées par } \\
\text { l'enseignant }\end{array}$ & 62 & $7 / 8$ \\
\hline
\end{tabular}

Le dispositif exercice écrit se caractérise par la présence massive de régulations locales initiées par l'enseignant. Cette présence est si massive qu'on peut dire que le dispositif exercice écrit engendre une forte participation des élèves, ce qui suscite des régulations de la part des enseignants.

\section{L'élève et la régulation}

Les régulations locales constituent des formats interactifs favorisant l'intervention des élèves. Nous voulons donc voir dans quelle mesure, sur quel dispositif les élèves participent, dans la prise de parole, à la construction de l'objet.

Si nous regardons d'un peu plus près la participation des élèves aux dialogues régulatifs maître /élèves (tableau ci-après), nous remarquons que dans le dispositif didactique « formulation de règle » ne comporte pas d'intervention d'élèves, ce dispositif apparaît uniquement dans le discours des enseignants (un discours magistral).

Pour solliciter l'intervention des élèves, certains enseignants donnent la réponse à moitié et attendent que les élèves terminent l'autre moitié.

Exemple

EB : ce texte c'est une fable très bien qui est l'auteur de ce texte Cette fable

est écrit par qui // est écrit PAR

EB : jean

és : jean de la fontaine

Nous remarquons que l'ensemble des régulations est davantage à l'initiative de l'enseignant. Quant aux obstacles régulés à l'initiative des élèves, nous constatons qu'ils sont quasi- inexistants. L'élève semble passif, sauf lorsque l'enseignant l'aide à participer. L'enseignant ne veut pas être détourné de l'objectif qu'il se serait fixé (fixé aussi par l'institution). Donc, il essaye 
Quand l'auteur se livre : du vécu à la création littéraire

Notes d'une musique ancienne de Salah Benlabed

d'avancer et de terminer sa leçon sans trop s'attarder sur les obstacles rencontrés, il régule mais sans pour autant perdre trop de temps quitte à faire une leçon magistrale (il explique, pose des question et c'est lui qui apporte les réponses). 
Camélia Nabila Bechiri

Tableau : régulations initiées par les élèves

\begin{tabular}{|c|c|c|c|c|c|}
\hline & \multirow[t]{2}{*}{ Régulation } & \multicolumn{3}{|l|}{ Type d'obstacle } & \multirow[b]{2}{*}{ Total } \\
\hline & & $\begin{array}{l}\text { Compréhension } \\
\text { d'un texte ou } \\
\text { une phrase }\end{array}$ & \begin{tabular}{|l|} 
Explication \\
de cours
\end{tabular} & \begin{tabular}{|l|} 
Formulation \\
de règle
\end{tabular} & \\
\hline \multirow{2}{*}{$\begin{array}{l}\text { Classe } \\
\text { de } \\
\text { monsieur } \\
\mathrm{T}\end{array}$} & $\begin{array}{l}\text { Initiées par } \\
\text { l'enseignant }\end{array}$ & 00 & 05 & 00 & 05 \\
\hline & $\begin{array}{l}\text { Initiés par } \\
\text { l'élève }\end{array}$ & 00 & 00 & 00 & 00 \\
\hline \multirow{2}{*}{$\begin{array}{l}\text { Classe } \\
\text { de } \\
\text { madame } \\
\text { A }\end{array}$} & \begin{tabular}{|l|} 
Initiées par \\
l'enseignant
\end{tabular} & |------------- & 02 & 00 & 02 \\
\hline & \begin{tabular}{|l|} 
Initiés par \\
l'élève \\
\end{tabular} & |------------- & 00 & 00 & 00 \\
\hline \multirow{2}{*}{\begin{tabular}{|l|} 
Classe \\
de \\
madame \\
$\mathrm{H}$ \\
\end{tabular}} & \begin{tabular}{|l|} 
Initiées par \\
l'enseignant
\end{tabular} & |------------- & 01 & 00 & 01 \\
\hline & $\begin{array}{|ll|}\begin{array}{l}\text { Initiés par } \\
\text { l'élève }\end{array} \\
\end{array}$ & |-------------- & 00 & 00 & 00 \\
\hline \multirow{2}{*}{$\begin{array}{l}\text { Classe } \\
\text { de } \\
\text { madame } \\
\text { O }\end{array}$} & \begin{tabular}{|l|} 
Initiées par \\
l'enseignant \\
\end{tabular} & |------------- & 00 & 00 & 00 \\
\hline & \begin{tabular}{|l|} 
Initiés par \\
l'élève
\end{tabular} & |------------- & 00 & 00 & 00 \\
\hline \multirow{2}{*}{\begin{tabular}{|l|} 
Classe \\
de \\
monsieur \\
B \\
\end{tabular}} & \begin{tabular}{|l|} 
Initiées par \\
l'enseignant \\
\end{tabular} & |------'----- & 00 & 00 & 00 \\
\hline & $\begin{array}{l}\text { Initiés par } \\
\text { l'élève }\end{array}$ & |------------- & 00 & 00 & 00 \\
\hline \multirow{2}{*}{\begin{tabular}{|l|} 
Classe \\
de \\
monsieur \\
B \\
\end{tabular}} & \begin{tabular}{|l|} 
Initiées par \\
l'enseignant
\end{tabular} & |-------------- & 02 & 00 & 02 \\
\hline & $\begin{array}{l}\text { Initiés par } \\
\text { l'élève }\end{array}$ & |-------------- & 00 & 00 & 00 \\
\hline \multirow{2}{*}{\begin{tabular}{|l|} 
Classe \\
de \\
monsieur \\
$\mathrm{S}$
\end{tabular}} & \begin{tabular}{|l|} 
Initiées par \\
l'enseignant
\end{tabular} & 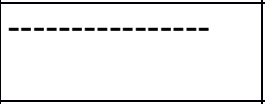 & 07 & 00 & 07 \\
\hline & \begin{tabular}{|l|} 
Initiés par \\
l'élève
\end{tabular} & \begin{tabular}{|l|}
---------------- \\
\end{tabular} & 00 & 00 & 00 \\
\hline \multirow{2}{*}{$\begin{array}{l}\text { Classe } \\
\text { de } \\
\text { monsieur } \\
\text { G } \\
\end{array}$} & \begin{tabular}{|l|} 
Initiées par \\
l'enseignant
\end{tabular} & 04 & 01 & 00 & 05 \\
\hline & \begin{tabular}{|l|} 
Initiés par \\
l'élève \\
\end{tabular} & 00 & 00 & 00 & 00 \\
\hline Total & \begin{tabular}{|l|} 
Initiées par \\
l'enseignant
\end{tabular} & 04 & 18 & 00 & 22 \\
\hline
\end{tabular}




\begin{tabular}{|l|l|l|l|l|}
\hline $\begin{array}{l}\text { Initiées par } \\
\text { l'élève }\end{array}$ & 00 & 00 & 00 & 00 \\
\hline
\end{tabular}

Le geste de régulation interactive fait partie du fil de l'action des enseignants et apparait tout au long de la construction de notre objet grammatical «types de phrase-formation d'adverbe.... ». En focalisant nos observations sur les trois dispositifs les plus régulés, nous pouvons observer comment l'objet grammatical se construit au fil de l'action dans un dialogue interactif entre enseignants et élèves.

Le dispositif « lecture et la compréhension du texte ou de phrase » pose certains problèmes spécifiques. En premier lieu, la lecture n'est pas facile pour les élèves au niveau de la prononciation; les élèves n'arrivent pas à lire correctement le texte. Des régulations de la part de l'enseignant sont entreprises mais juste pour améliorer la prononciation. Pour ce qui est du dispositif de compréhension, le contenu du texte paraît complexe aux élèves. Les enseignants semblent éviter ce dispositif : seulement deux enseignants s'y aventurent. La compréhension de textes utilisés par certains enseignants fait l'objet de nombreuses régulations. Dans une seule pratique observée, enseignants et élèves créent du sens autour de la lecture du texte particulier et tentent d'identifier les verbes à l'impératif présent impératif. La construction de l'objet grammatical provoque plusieurs événements régulatifs, initiés par l'enseignant, afin de l'expliquer et d'extraire une règle. À leur tour, les élèves essayent tant bien que mal de participer mais cette participation reste minime, sans l'intervention de l'enseignant, la leçon n'avancera pas.

De nombreuses régulations dans le dispositif exercice écrit, plutôt à l'initiative de l'enseignant, sont aussi mises en œuvre pour essayer de consolider les acquis des élèves. Dans leur participation, il nous semble que les élèves donnent des réponses de façon aléatoire et, selon la réaction de l'enseignant ils changent rapidement de réponse, sinon c'est l'enseignant qui se charge de corriger. Les élèves participent souvent en groupe.

Conclusion

Pour conclure, le geste de régulation interactive est très présent dans toutes les séances observées. Par rapport à notre objet "grammaire », les obstacles les plus régulés sont de plusieurs types mais nous retiendrons les problèmes posés par l'objet grammaire : d'une part, les élèves ont du mal à lire et comprendre le texte ou les phrases utilisées comme exemple. D'autre part, certains objets grammaticaux nécessitent d'autres connaissances (la transformation de la voix passive à la voix active nécessite la connaissance des temps verbaux et de l'accord du participe passé). Ils sont donc plus difficiles à expliquer car cela prend du temps et l'enseignant n'a qu'une heure pour réaliser sa leçon de grammaire. La mise en pratique dans des exercices de l'objet grammaire suscite de nombreuses régulations. Mais nous pensons que les 
variables didactiques susceptibles de provoquer des régulations, des adaptations faisant avancer la construction du savoir sont inadaptées.

L'analyse des régulations locales a permis de constater que les pratiques du passé perdurent et influencent le déroulement de l'enseignement. Dans cette perspective, l'élève se voit conférer un rôle de spectateur passif.

\section{Références bibliographiques :}

(1)- Schneuwly, B. \& Bain, D. (1993). Mécanismes de régulation des activités textuelles:

(2)- Stratégies d'intervention dans les séquences didactiques. Dans L. Allal, D. Bain \& P.

(3)- Perrenoud. : Évaluation formative et didactique du français. Neuchâtel : Delachaux et Niestlé.

(4)- Schneuwly, B. (2009a). "L'objet enseigné ». Dans Schneuwly,B \& Dolz ,J (Eds). Des objets enseignés en classe de français: Le travail de l'enseignant sur la rédaction de textes argumentatifs et sur la subordonnée relative. Rennes: PU. (5)- Schneuwly, B. (2009b). "Le travail enseignant». Dans Schneuwly,B \& Dolz,J (Eds). Des objets enseignés en classe de français: Le travail de l'enseignant sur la rédaction

(6)- Sensevy, G. (2007). Des catégories pour décrire et comprendre l'action didactique. Dans Sensevy, G \& Mercier,A (Eds), Agir ensemble . Rennes: PU . (7)- Tisset, C. (2005). Observer, Manipuler,Enseigner la langue au cycle 3.Paris : Hachette Education .

(8)- Vygotski, L.-S. (1985). Pensée et langage suivi de Commentaires sur les remarques critiques de Vygotski par J. Piaget, Messidor, Paris : Éditions sociales. (9)- Vygotski, L.-S. (1934 /1997). Pensée et langage. Paris : Éditions sociales . (10)- Textes institutionnels

(11)- Roegiers, Xavier, (2006) : L'approche par compétences dans l'école algérienne, éd. ONPS (Office national des publications scolaires), Alger, novembre, sous la réalisation du Ministère de l'Education Nationale et du Programme d'appui de L'UNESCO à la réforme du système éducatif (PARE). 